\title{
Team Sweden
}

\author{
M. Boman ${ }^{2}$, K. LeBlanc ${ }^{1}$, C. Guttmann ${ }^{2}$, and A. Saffiotti ${ }^{1}$ \\ 1 AASS, Center for Applied Autonomous Sensor Systems \\ Dept. of Technology and Science, Örebro University \\ Fakultetsgatan 1, S-701 82 Örebro, Sweden \\ 2 DSV, Department of Computer and Systems Sciences \\ Stockholm University and The Royal Institute of Technology \\ Electrum 203, S-164 40 Kista, Sweden
}

\section{Introduction}

"Team Sweden" is the Swedish national team that entered the Sony legged robot league at the RoboCup 99 competition. We had two main requirements in mind when preparing our entry to the competition:

1. The entry should effectively address the specific challenges present in this domain; in particular, it should be able to tolerate errors and imprecision in perception and execution; and

2. it should illustrate our research in autonomous robotics, by incorporating general techniques that can be reused in different robots and environments.

While the first requirement could have been met by writing some ad hoc competition software, the second one led us to develop principled solutions that drew upon our current research, and that pushed it further ahead.

\section{Team Development}

The work has been distributed over several universities in Sweden, which has made the project organization especially demanding. The three main cities of activity were Stockholm, Örebro, and Ronneby, which are separated by a geographical distance of up to $600 \mathrm{Km}$.

Team Leader: Magnus Boman (Stockholm)

Team Members: in addition to the authors of this paper, the team included:

- from Stockholm: M. Ericmats (MSc student), J. Kummeneje (PhD student), and A. Tollet (MSc student);

- from Örebro: M. Karlström (undergrad), D. Petersson (undergrad), and Z. Wasik (PhD student);

- from University of Karlskrona/Ronneby: P. Davidsson (ass. professor), M. Fredriksson (PhD student), and S. Johansson (PhD student).

Many other people contributed to the team in several ways. Their names are listed in the team web page.

Web page: http://www.dsv.su.se/ robocup/teamsweden/. Information on the current progress of the team: http://aass.oru. se/Living/RoboCup/. 


\section{Robot Architecture}

Each robot was endowed with a layered architecture for autonomy inspired by the Thinking Cap, ${ }^{1}$ sketched in Fig. 1 . The lower layer provides an abstract interface to the physical functionalities of the robot. The middle layer is responsible for maintaining a local representation of the space around the robot (PAM), and for implementing a set of robust tactical behaviors (HBM). The higher layer maintains a global map of the field (GM) and makes real-time strategic decisions (RP). More detailed information can be found at the Team web sites.

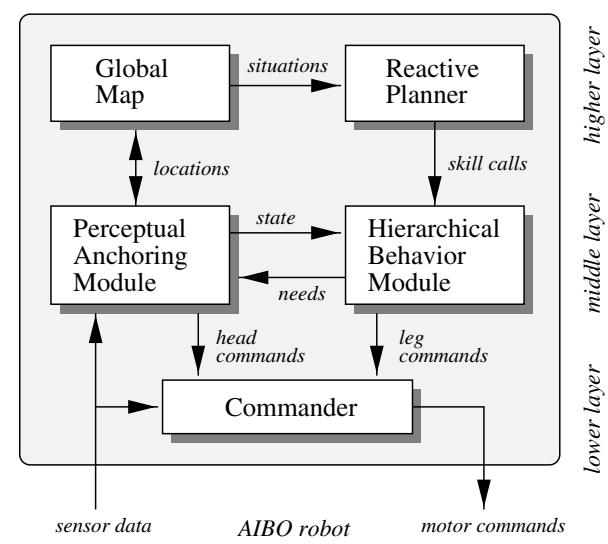

Fig. 1. The variant of the Thinking Cap architecture used at RoboCup '99.

\section{Perception}

The main locus of perception is the PAM, which acts as a short term memory of the location of the objects around the robot. The position of each object is updated by three mechanisms: by perceptual anchoring, whenever the object is detected by the camera; by global information - for the fixed objects onlywhenever the robot registers its own location in the global map; and by odometric clamping, whenever the robot moves.

Each object is associated with two fuzzy predicates, that take truth values in the $[0,1]$ interval: the anchored predicate measures how much the object's position is supported by recent perception; the needed predicate measures how much that object is needed by the currently running behaviors. These are used to measure how much it is important to re-acquire a given object $o$ by

$$
\operatorname{important}(o)=\operatorname{needed}(o) \cdot(1-\operatorname{anchored}(o)) \text {. }
$$

We use importance to decide the perceptual focus by selecting the object $f$ with the highest importance. (We need focus control because we have only one

\footnotetext{
${ }^{1}$ The agent architecture based on fuzzy logic in use at Örebro University. This is a successor of the architecture originally developed for the robot Flakey [1].
} 
camera, and many potential objects of interest.) The camera is pointed to the expected position of $f$, and a head scan is done if the object is not found. The use of (1) results in selecting all of the needed objects in a round-robin fashion.

Object recognition relies on the color detection hardware in the Sony robot, to which we provide the intended color signatures (produced off-line from color samples). We combine color blobs into features by a model-based approach: for instance, a green blob and a pink blob in a given geometric relation are fused into a landmark feature. These features are checked against the three criteria illustrated in Fig. 2. First, we reject features which are "astray": e.g., the pink band of a landmark cannot be a ball feature since it does not lay on the ground. Second, we select features which are in the "anchoring zone," a small fovea in the image where we can reliably measure the object's position. The remaining features are consider to be in "tracking zone," meaning that they should be brought into the anchoring zone by moving the camera.
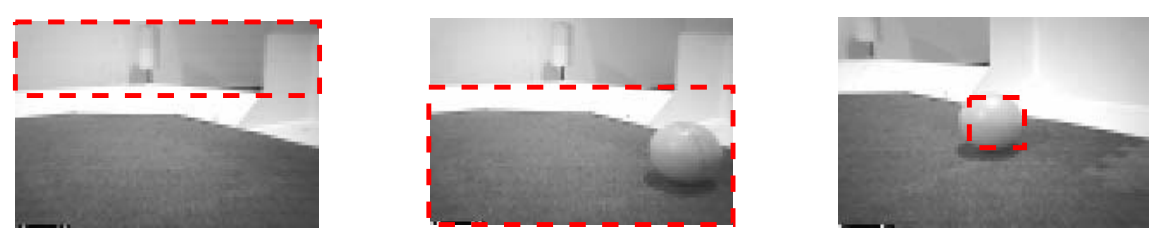

Fig. 2. A ball feature in the astray (left), tracking (center), and anchoring zone (right).

\section{Control}

The HBM implements a set of motion behaviors realized using fuzzy logic techniques and organized in a hierarchical way. The use of fuzzy logic brings two main advantages: firstly, fuzzy behaviors can tolerate some amount of uncertainty in the position estimates; secondly, it is easy to write complex control strategies using a simple rule based language [2]. As an illustration, the following set of fuzzy rules implement the "GoToPosition" behavior.

$\begin{array}{ll}\text { IF (AND (NOT (PositionHere), PositionLeft)) } & \text { TURN (LEFT); } \\ \text { IF (AND (NOT PositionHere), PositionRight)) } & \text { TURN (RIGHT); } \\ \text { IF (OR(PositionHere, PositionAhead)) } & \text { TURN (AHEAD); } \\ \text { IF (AND(NOT(PositionHere), PositionAhead)) } & \text { GO (FAST); } \\ \text { IF (OR(PositionHere, NOT(PositionAhead))) } & \text { GO (STAY); }\end{array}$

The fuzzy predicates in the antecedents rely on the information in the PAM; 'GO' and 'TURN' are fuzzy set-points for linear and rotational velocity, respectively. These are passed to the Commander module, which translates them to an appropriate walking style. The translation simplifies the writing of motion behaviors, and makes them more portable between different platforms. It also allows us to easily modify the walking style used to implement each velocity pair.

Simple behaviors can be composed into increasingly more complex behaviors by using fuzzy rules that activate concurrent sub-behaviors. For instance, the following rules implement the "GoAndScore" behavior. 


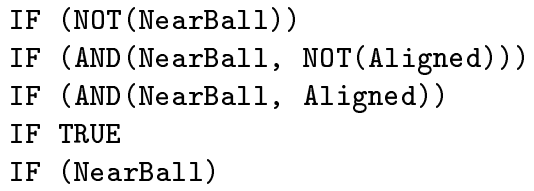

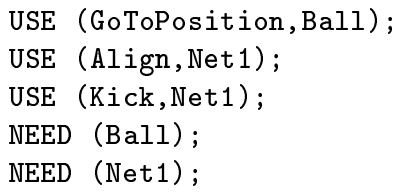

These rules also show how behaviors communicate the current perceptual needs to the PMA via the 'NEED' keyword. For instance, when the ball is at $400 \mathrm{~mm}$ from the robot the truth value of "NearBall" is 0.7 , and the above rules assert a value of needed of 1.0 for the object Ball and of 0.7 for Net1. These values are used by the gaze control mechanism to track the relevant features.

\section{Localization}

Spatial information is represented at two levels: locally, in the PAM; and globally, in the GM. The GM incorporates prior knowledge of the relative position of the fixed objects in the field. The information in the PAM and in the GM is registered by estimating the robot's posture in the field using the observed position of the landmarks. Unfortunately, we could not test this mechanism to a sufficient extent by time of the competition, so we only used local information.

\section{Strategy}

We used hierarchical behavior composition to write simple fixed strategies like "GoAndKick." More complex strategies are dynamically generated by the RP, which implements a decision making scheme inspired by cognitive psychology [3]. The RP decides the top-level behavior to activate by a voting mechanism, where votes correspond to motivations for using a given behavior in a given situation. The RP was not fully developed by the time of the competition, so we only used very basic strategies for the players and for the goal-keeper.

\section{Conclusion}

Our experience at RoboCup '99 has shown that the general principles and techniques developed in our research could be successfully applied to a radically different domain. Fuzzy logic proved beneficial in writing robust behaviors, and in developing an effective gaze control strategy. We believe that fuzzy logic could also bring substantial advantages in self localization and in flexible reactive planning. We plan to explore these issues in preparing our entry to RoboCup '2000. Acknowledgements We thank Aline Dahlke at Sony France and the Sony staff in Japan for being so understanding of the difficulties of our distributed work situation. Founding was provided by the Universities of Örebro, of Stockholm, and of Ronneby.

\section{References}

1. A. Saffiotti, K. Konolige, and E.H. Ruspini. A multivalued-logic approach to integrating planning and control. Artificial Intelligence, 76:481-526, 1995.

2. A. Saffiotti. Using fuzzy logic in autonomous robot navigation. Soft Computing, 1(4):180-197, 1997. Online at http://aass.oru.se/Living/FLAR/.

3. C. Guttmann A software architecture for four-legged robots. M.Sc. Thesis, Dept. of Computer and System Science, Stockholm University, 1999. 